\title{
Research on the Application of Physical Fitness Monitoring Data Intelligent Management to Guide Physical Education Teaching
}

\author{
LIU Dongxu ${ }^{1, a}$, LI Jiayi ${ }^{2, b, *}$ \\ ${ }^{1}$ Department of Physical Education, Jilin Institute of Chemical Technology, Jilin Jilin China \\ ${ }^{2}$ College of Information and Control Engineering, Jilin Institute of Chemical Technology, Jilin Jilin China \\ a85169593@qq.com, ${ }^{b}$ washy59@sina.com, \\ *Corresponding author
}

\begin{abstract}
This paper aims at the problems that need to be solved urgently in physical education teaching. With the help of artificial intelligence technology and the rapid development of 5G era, wearable devices are used to monitor students' classroom status in real time and to statistically process the monitoring data. The experimental results show that the intelligent management of physique monitoring data can optimize classroom efficiency, save teachers' time to acquire and process classroom data, realize early warning of classroom sports risks, reduce classroom sports risks, respect individual differences, and realize one-to-one understanding and guidance.
\end{abstract}

Keywords: physical education, intelligent management of data, physical fitness monitoring

\section{体质监测数据智能管理指导体育教学的应用研究}

\author{
刘东旭 $1, \mathrm{a}$, 李佳怡 $2, \mathrm{~b}, *$
}

1 吉林化工学院体育教学部, 吉林, 吉林, 中国

2 吉林化工学院信息与控制工程学院, 吉林, 吉林, 中国

a85169593@qq.com

b*washy59@sina.com

\section{摘要}

本文针对体育教学中叒待解决问题入手，借助人工智能技术以及 $5 \mathrm{G}$ 时代的迅速发展，利用可穿戴设备 对学生课堂状态进行实时监测并对监测数据进行统计处理。实验结果指出, 体质监测数据智能管理能 够优化课堂效率，节省教师获取和处理课堂数据的时间，能够对课堂运动风险实现预警，降低课堂运 动风险, 同时能够尊重个体化差异, 实现一对一的了解和指导。

关键词: 体育教学; 数据智能管理; 体质监测

\section{1. 前言}

随着近年来校园体育中学生在参加体育活动的过 程中危险状况频发, 学者对于可穿戴运动监测设备、运 动 APP 的研究也逐步深入, 从研究中可以发现, 在学生 参加体育活动的过程中进行有效的监测评价, 可以更清 楚的了解自身的各项物理和生理指标, 通过对心率的合 理控制, 达到合理的锻炼负荷。目前对于可穿戴设备以 及运动 APP 的研究很多, 但都没有真正意义的将两者相 结合, 实现数据采集后的智能化管理, 如何能让两者有 机的结合, 应用到体育教学活动当中去, 是我们需要深
入去研究的问题。

\section{2. 国内外相关研究的学术史梳理及研究动态}

近年来大学生大多成为了 “低头族”, 对于体育活 动的参与极为缺乏, 因此大学生身体素质每况愈下, 针 对这一现象, 我国加大了对学生体质健康测试的力度, 对于大学生各类体育运动都在提高重视 ${ }^{[1]}$ 。但是, 基于 学生在体育活动中时常会出现危险情况, 体育教师无法 对每个学生的身体状态完全掌握, 所以对于学生在体育 活动中的身体状态监测显得十分重要, 这是学校目前对 于学生开展体育活动亟待解决的问题。人工智能是一门 


\section{3. 研究价值}

学、哲学等多种学科基础上发展起来的, 能够模拟、眼 神、扩展人类智能的学科 ${ }^{[2]}$ 。随着计算机技术的高速发 展, 人工智能技术也得到了极大的提高, 其在体育领域 中的应用也更加的广泛。

\section{1. 国内研究现状}

在 “互联网+”影响下, 全民健身活动正向智能运 动、科学运动、互联网化的方向发展, 以应对人们在自 身健康指标、运动塑身塑形等细分层次上的更高诉求。 可穿戴设备的兴起及运动器械的智能化, 不仅给全民健 身运动带来了科学指导, 而且改变着人们的健康生活和 运动轨迹 ${ }^{[3]}$ 。

随着近年来校园体育中学生在参加体育活动的过 程中危险状况频发, 国内学者对于可穿戴运动监测设备、 运动 APP 的研究也逐步深入, 从研究中可以发现, 在学 生参加体育活动的过程中进行有效的监测评价, 可以更 清楚的了解自身的各项物理和生理指标, 通过对心率的 合理控制, 达到合理的锻炼负荷。目前国内对于可穿戴 设备以及运动 APP 的研究很多, 但都没有真正意义的将 两者相结合, 实现数据采集后的智能化管理, 如何能让 两者有机的结合, 应用到体育教学活动当中去, 是我们 需要深入去研究的问题。

\section{2 国外研究现状}

目前, 人工智能在国际比赛的应用已经较为普及, 追求 “更快、更高、更强”, 提升竞技水平和专业化水 平, 既是竞技体育发展的重要目标, 也是竞技体育的鬼 力所在。而人工智能则为其竞技水平和专业化水平提供 了重要的手段与方法。

在专业训练方面, 通过人工智能对训练、备战和比 赛情况等海量数据进行处理和大数据分析, 教练员可以 适时调整策略和排兵布阵, 运动员能够更好、更有效地 训练, 从而提升运动员及球队的技术水平, 优化运动员 的实时表现和场上状态, 谋取更佳成绩 ${ }^{[4]}$ 。

从竞赛组织看, 在赛场上, 运动员与裁判员之间可 能因误判而产生矛盾, 而人工智能的运用可以有效规避 这些问题。网球比赛应用 “鹰眼技术”, 不仅把赛场分 割成以毫米计算的若干测量单位, 把网球飞行轨迹的数 据生成三维图像, 在大屏幕上呈现出网球的具体飞行轨 迹及落点, 而且从数据采集到结果演示的总耗时不超过 10 秒, 误差在 $1 \%$ 以下, 为裁判员进行精准判罚提供了 重要帮助 ${ }^{[5]}$ 。

综上所述, 结合 AI 技术支持, 完成对大学生在体 育活动中的实时体质监测, 能够清楚的掌握其身体锻炼 情况, 并对取得数据进行智能化管理, 从而调整运动负 荷, 避免危险事件的发生。对于体育教师来讲, 对于取 得的数据的智能化管理, 能够追踪其锻炼习惯特点, 从 而更全面的掌握其身体情况，针对性的给与课内外指导。

\section{1 学术价值}

对大学生在体育活动的过程中的身体状态进行监 测采集以及智能化管理, 能促进运动锻炼习惯的形成, 对运动进行实时的监测, 提供科学的运动方案, 减少运 动风险的发生, 能为健康生活提供支持和服务, 同时也 能为先进科学技术促进体育教学优化研究提供一定的 理论依据 ${ }^{[6]}$ 。

\section{2 应用价值}

使用可穿戴设备对运动进行监测, 能提升监测水平、 增加监测项目、减轻运动负担, 与手机 APP 或电脑相 结合, 能增加多种功能, 增加使用积极性和长久性。数 据智能管理系统和可穿戴设备相结合, 能使大学生以更 快、更方便的方式, 进行更加科学的、合理的运动, 大 学生可以不受时间和场地的限制, 进行多种多样的体育 活动。而智能设备和可穿戴设备的有机结合, 能帮助体 育教师在一定程度上监控运动强度和负荷, 减少了运动 的盲目性, 提高运动安全性, 并能提高锻炼效率和兴趣, 增强趣味性, 使运动更加合理。

\section{4. 研究目标}

大学生体质监测智能采集系统设计, 以可穿戴设备 为基础, 监测大学生在体育活动中的运动物理指标 (计 步、运动定位) 和生理指标的监控 (心率); 另外根据 这些数据简单计算卡路里的消耗、运动速度、距离、运 动轨迹的记录功能, 并以心率监测为核心, 通过运动过 程的心率监测, 控制运动强度, 并能根据运动中、运动 后心率变化和其他 (问答) 对运动过程中产生的数据进 行智能管理, 使体育教师和学生了解此次运动情况, 为 下次运动做一定的参考。在设计和实现过程中紧紧围绕 以上内容对可穿戴设备和 数据管理系统设计和实现, 最终完成智能数据采集系统, 并将个体数据形成数据曲 线及可调用的历史数据包, 为使用者在体育教学和自主 锻炼中起到指导和帮助作用。

\section{5. 研究总体框架}

体质监测数据智能管理系统研究总体框架如图一 所示。 


\section{3 研究对象的体质数据监测应用}

课题以在校大一大二体育与健康课程学生为基本 研究对象, 选取实验班和对照班各 30 名男同学, 在实 验前对研究对象进行前测指标对比, 结果表明, 两班学 生在监测指标上并无明显差异, 同时实验班和对照班都 采取同样的教学内容和教学计划, 两班教师执教水平相 当, 符合实验要求。实验过程为十次课, 实验班进行体 质监测管理, 对照班按照传统模式进行教学, 对两班在 教学过程中身体状态、教学数据获取和处理进行比对。

结果表明, 实验班与对照班在实验后的监测指标无 明显差异, 但在课堂过程中实验班因为能够获取更多学 生的身体指标, 教师根据指标安排心率过高学生降低运 动强度和负荷 15 人次, 同时将心率较平稳的学生增加 运动强度和负荷 10 人次, 实现的危险的预警和教学的 个性化指导, 对比对照班, 由于教师获取学生指标较少, 只能采取抽查和根据教学经验判断, 对比实验班教师对 课堂的掌控就明显不足。

在对课堂数据的获取和处理上, 对照班由于采取传 统的教学方法, 教师沿用纸笔秒表的数据记录方法, 获 取数据的种类有限的情况下, 在统计一个班的教学数据 平均用时增加 30 分钟的时间, 教学效率较低。实验班 教师能够借助监测智能管理系统实时监测学生身体状 态和运动指标的同时, 数据上传至系统并进行处理运算, 教师只需简单的手机和 PC 端操作, 就能够查看每个学 生的历史数据。

\section{7. 结论}

实验结果表明, 体质监测数据智能管理系统能够有 效指导体育教学, 对体育教学过程中运动风险形成主动 预警, 降低发生运动风险的几率。同时教师在体育教学 中能够有效控制学生的运动负荷和强度, 避免仅凭经验 判断, 做到有理有据。在课堂数据的获取和统计上, 系 统也能够提高教学效率, 减轻教师教学负担并提升课堂 的先进性。综上所述, 体育教学中对学生进行体质监测 数据智能管理, 能够指导教师少走弯路, 优化课程, 减 少弊端, 形成科学、有效、安全的教学环境。

\section{REFERENCES}

[1] He Maolin. Research on the current situation and Countermeasures of physical exercise attitude and behavior of college students in Shijiazhuang City [D]. Hebei Normal University, 2016

[2] Zuo Xi, Qi Cheng. Using modern educational technology to develop vocational education [J]. Forum on industry and science and technology, 2013,12 (01): 167

[3] Yu Jianming. The current situation of College Students' Sports Participation under the background of "Internet plus" [D]. Shanghai Institute of Physical Education, 2017.
用要求, 系统各项参数正常, 运行平稳, 能够满足体育 教学各项指标的采集、记录、运算和储存。 
[4] Yang Xiaofan, Guo Yaping. Research on the development trend of sports wearable equipment [J]. Fujian sports science and technology, 2018,37 (03): 17-19 $+37$

[5] Zhu Yihong. Analysis of the influence of artificial intelligence on Sports Development [C]. Chinese Academy of Sports Sciences. Summary of papers of the 11th National Congress of Sports Sciences. Chinese Academy of Sports Sciences: Chinese Academy of Sports Sciences, 2019:4234-4235

[6] Guo Chengzhi. Research on the design and implementation of sports fitness monitoring app [D]. Guangzhou Institute of physical education, 2017

[7] Liu Dongxu. Design of Data Acquisition System for Fitness Test Based on Wearable Equipment [J]. Digital Technology and Application, 2019,37(12):155+157 